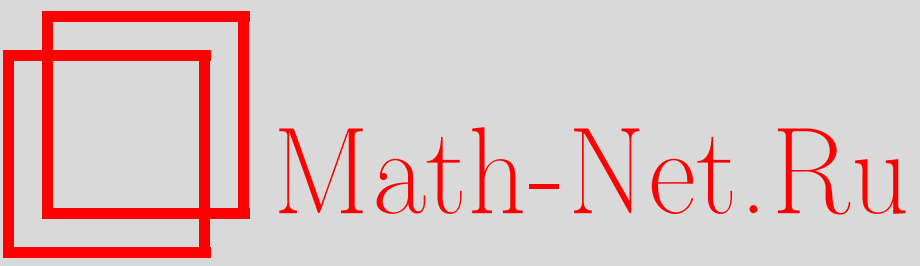

В. Ф. Формалев, И. А. Селин, Е. Л. Кузнецова, Моделирование тепловых волн в нелинейном анизотропном пространстве, Вестн. Сам. гос. техн. ун-та. Сер. Физ.-мат. науки, 2010, выпуск 1(), 239-243

DOI: https://doi.org/10.14498/vsgtu745

Использование Общероссийского математического портала Math-Net.Ru подразумевает, что вы прочитали и согласны с пользовательским соглашением

http: //www. mathnet.ru/rus/agreement

Параметры загрузки:

IP : 54.237 .206 .68

26 апреля 2023 г., 12:28:07 
УДК 517.958+536.2(075)

\section{МОДЕЛИРОВАНИЕ ТЕПЛОВЫХ ВОЛН В НЕЛИНЕЙНОМ АНИЗОТРОПНОМ ПРОСТРАНСТВЕ}

\section{В. Ф. Формалёв, И. А. Селин, Е. Л. Кузнецова}

Московский авиационный институт (государственный технический университет), 125993, Москва, А-80, ГСП-3, Волоколамское шоссе, 4.

E-mails: soll06@bk.ru, priem@mai.ru

Получено аналитическое решение краевой задачи с граничным условием первого рода в начале координат для квазилинейного уравнения параболического типа в анизотропном пространстве со степенной зависимостью компонентов тензора теплопроводности от температурь. Анализ решения показал волновой характер распространения тепла в анизотропном пространстве с конечной скоростью в отличие от бесконечной скорости для линейного уравнения параболического типа. Установлено, что фронт тепловой волны в анизотропном пространстве имеет форму эллипсов на плоскости. Выполнен анализ результатов.

Ключевые слова: квазилинейное параболическое уравнение, тепловая волна, тензор теплопроводности, температура, тепловой поток, фронт.

Введение. Известно, что распространение тепла, описываемое линейным уравнением параболического типа, осуществляется с бесконечной скоростью [1-5], что следует из любого фундаментального решения линейного уравнения диффузии. Этот факт приводит к парадоксу распространения тепла, поскольку все опытные данные показывают хотя и большую, но конечную скорость распространения тепла. Для преодоления этого парадокса ещё Максвелл выдвинул гипотезу о том, что распространение тепла имеет не только диффузионный, но и волновой характер.

В данной статье рассматривается краевая задача для квазилинейного уравнения теплопроводности в анизотропном пространстве и устанавливается волновой характер распространения тепла, то есть в каждый момент времени изотермическая поверхность разделяет конечное пространство с ненулевой температурой и полубесконечное пространство с нулевой (начальной) температурой.

1. Постановка задачи. Рассматривается нестационарное распределение температуры $T(x, y, t)$ в двумерном анизотропном пространстве при действии источника температуры $T_{0}$ в начале координат $O(0,0)$, то есть рассматривается следующая начально-краевая задача при $(x, y) \in \mathbb{R}^{2}, t>0$ :

$$
\begin{gathered}
c \rho \frac{\partial T}{\partial t}=\frac{\partial}{\partial x}\left(\lambda_{x x}(T) \frac{\partial T}{\partial x}\right)+\frac{\partial}{\partial x}\left(\lambda_{x y}(T) \frac{\partial T}{\partial y}\right)+ \\
+\frac{\partial}{\partial y}\left(\lambda_{y x}(T) \frac{\partial T}{\partial x}\right)+\frac{\partial}{\partial y}\left(\lambda_{y y}(T) \frac{\partial T}{\partial y}\right) ; \\
T(0,0, t)=T_{0}, \\
T(x, y, 0)=0 .
\end{gathered}
$$

Компоненты тензора теплопроводности определяются выражениями [6]

$$
\lambda_{x x}(T)=\lambda_{\xi}(T) \cos ^{2} \varphi+\lambda_{\eta}(T) \sin ^{2} \varphi, \quad \lambda_{y y}(T)=\lambda_{\xi}(T) \sin ^{2} \varphi+\lambda_{\eta}(T) \cos ^{2} \varphi,
$$

Владимир Федорович Формалёв (д.ф.-м.н., проф.), профессор, каф. вычислительной математики и программирования. Илья Александрович Селин, аспирант, каф. вычислительной математики и программирования. Екатерина Лъвовна Кузнецова (к.ф.-м.н., доц.), докторант, каф. вычислительной математики и программирования. 


$$
\lambda_{x y}(T)=\lambda_{y x}(T)=\left(\lambda_{\xi}(T)-\lambda_{\eta},(T)\right) \sin \varphi \cos \varphi,
$$

где $\lambda_{\xi}(T)=k_{\xi} T^{\sigma}, \lambda_{\eta}(T)=k_{\eta} T^{\sigma} ; k_{\xi}, k_{\eta}$ - const; $\varphi$ - угол, ориентирующий главную ось $O \xi$ и ось $O \eta$ относительно декартовой оси $O x$.

Таким образом, необходимо найти нестационарное распределение температур $T(x, y, t)$ под действием точечного источника $(2)$, приложенного в точке $O$.

2. Метод решения. Поворотом вокруг начала координат на угол $\varphi$ декартовой системы координат $x=\xi \cos \varphi-\eta \sin \varphi, y=\xi \sin \varphi+\eta \cos \varphi$ задача (1)-(3) сводится к задаче для уравнения, не содержащего смешанных дифференциальных операторов при $(\xi, \eta) \in \mathbb{R}^{2}, t>0$ :

$$
\begin{gathered}
c \rho \frac{\partial T}{\partial t}=\frac{\partial}{\partial \xi}\left(k_{\xi} T^{\sigma} \frac{\partial T}{\partial \xi}\right)+\frac{\partial}{\partial \eta}\left(k_{\eta} T^{\sigma} \frac{\partial T}{\partial \eta}\right) ; \\
T(0,0, t)=T_{0}, \quad T(\xi, \eta, 0)=0 .
\end{gathered}
$$

Переходя к новой системе координат $x_{1}=\xi\left(b / k_{\xi}\right)^{1 / 2}, x_{2}=\eta\left(b / k_{\eta}\right)^{1 / 2}$, где $b$-любое число (например, $b=1$ ), получаем

$$
\begin{gathered}
\frac{\partial T}{\partial t}=a \frac{\partial}{\partial x_{1}}\left(T^{\sigma} \frac{\partial T}{\partial x_{1}}\right)+a \frac{\partial}{\partial x_{2}}\left(T^{\sigma} \frac{\partial T}{\partial x_{2}}\right) \\
T(0,0, t)=T_{0}, \quad T\left(x_{1}, x_{2}, 0\right)=0
\end{gathered}
$$

Будем искать решение задачи (4), (5) в автомодельном виде с использованием подстановки

$$
T\left(x_{1}, x_{2}, t\right)=t^{\alpha} \theta\left(q_{1}, q_{2}\right)
$$

где

$$
q_{1}=x_{1} / t^{\beta}, \quad q_{2}=x_{2} / t^{\beta} .
$$

В (6), (7) $\alpha$ и $\beta$ подлежат определению. Для их определения подставим (6), (7) в $(4),(5)$, получим

$$
\begin{aligned}
t^{\alpha-1}\left[\alpha \theta\left(q_{1}, q_{2}\right)-\beta\left(q_{1} \frac{\partial \theta}{\partial q_{1}}+q_{2} \frac{\partial \theta}{\partial q_{2}}\right)\right]= & \\
& =t^{\alpha(\sigma+1)-2 \beta} a\left[\frac{\partial}{\partial q_{1}}\left(\theta^{\sigma} \frac{\partial \theta}{\partial q_{1}}\right)+\frac{\partial}{\partial q_{2}}\left(\theta^{\sigma} \frac{\partial \theta}{\partial q_{2}}\right)\right] \\
t^{\alpha} \theta(0,0) & =T_{0}, \quad t^{\alpha} \theta\left(q_{1}, q_{2}\right)=0 .
\end{aligned}
$$

Из (8), (9) получаем

$$
\alpha=0, \quad \beta=1 / 2 .
$$

С учётом (10) задача $(8),(9)$ запишется при $\left(q_{1}, q_{1}\right) \in \mathbb{R}^{2}$ в виде

$$
\begin{gathered}
a \frac{\partial}{\partial q_{1}}\left(\theta^{\sigma} \frac{\partial \theta}{\partial q_{1}}\right)+a \frac{\partial}{\partial q_{2}}\left(\theta^{\sigma} \frac{\partial \theta}{\partial q_{2}}\right)+\frac{1}{2}\left(q_{1} \frac{\partial \theta}{\partial q_{1}}+q_{2} \frac{\partial \theta}{\partial q_{2}}\right)=0 \\
\theta(0,0)=T_{0} .
\end{gathered}
$$

Пусть функция $\theta\left(q_{1}, q_{2}\right)$ является радиально симметричной, т. е. $\theta\left(q_{1}, q_{2}\right)=\theta\left(q_{1}(r)\right.$, $\left.q_{2}(r)\right)=\tilde{\theta}(r)$, тогда задача $(11),(12)$ трансформируется в следующую задачу Коши для нелинейного обыкновенного дифференциального уравнения:

$$
2 a \frac{d}{d r}\left(\tilde{\theta}^{\sigma} \frac{d \tilde{\theta}}{d r}\right)+r \frac{d \tilde{\theta}}{d r}=0
$$




$$
\tilde{\theta}(0)=T_{0}, \quad \tilde{\theta}^{\sigma} \tilde{\theta^{\prime}}(0)=0 .
$$

Первый интеграл уравнения (13) записывается в виде

$$
\tilde{\theta}^{\sigma} \tilde{\theta}^{\prime}+\frac{r}{2 a} \tilde{\theta}=C_{1},
$$

причём в силу симметрии при $r=0$ постоянная $C_{1}=0$. Следовательно,

$$
\begin{gathered}
\tilde{\theta}^{\sigma} \tilde{\theta}^{\prime}+\frac{r}{2 a} \tilde{\theta}=0 ; \\
\tilde{\theta}(0)=T_{0} .
\end{gathered}
$$

Уравнение (14) является обыкновенным дифференциальным уравнением с разделяющимися переменными. Его общее решение имеет вид

$$
\tilde{\theta}(r)=\left(C_{2}-\frac{\sigma r^{2}}{4 a}\right)^{1 / \sigma}
$$

в котором постоянная интегрирования $C_{2}$ может быть определена из краевого условия (15):

$$
\tilde{\theta}(r)=\left(T_{0}^{\sigma}-\frac{\sigma r^{2}}{4 a}\right)^{1 / \sigma} .
$$

Возвращаясь к декартовым координатам, из (16) получаем решение исходной задачи (1)-(3):

$$
T(x, y, t)=\left[T_{0}^{\sigma}-\frac{\sigma}{4 a t}\left((x \cos \varphi+y \sin \varphi)^{2} \frac{\lambda}{k_{\xi}}+(-x \sin \varphi+y \cos \varphi)^{2} \frac{\lambda}{k_{\eta}}\right)\right]^{1 / \sigma} .
$$

3. Анализ результатов. На рис. 1-3 приведены результаты расчётов температурных полей по формуле (17) со следующими данными: $\sigma \in\{0,5 ; 1 ; 1,5\}, \varphi=0, k_{\xi}=5$, $k_{\eta}=1, b=1, c \rho=2000$ Дж $/ \mathrm{m}^{3} \cdot \mathrm{K}, T_{0}=5$.

Из представленных рисунков видно, что областями ненулевого решения в различные моменты времени являются области, при $\varphi=0$ ограниченные эллипсами с полуосями $l_{x}=\sqrt{T_{0}^{\sigma} \frac{4 a t}{\sigma} \frac{k_{\xi}}{b}}$ по оси $O x, l_{y}=\sqrt{T_{0}^{\sigma} \frac{4 a t}{\sigma} \frac{k_{\eta}}{b}}$ по оси $O y$. Температурное возмущение существует только внутри этих эллипсов.

Из рисунков следует, что распределение температур имеет волновой характер с фронтом волны на изотерме $T=0$, описываемой эллипсами с полуосями $l_{x}, l_{y}$, а
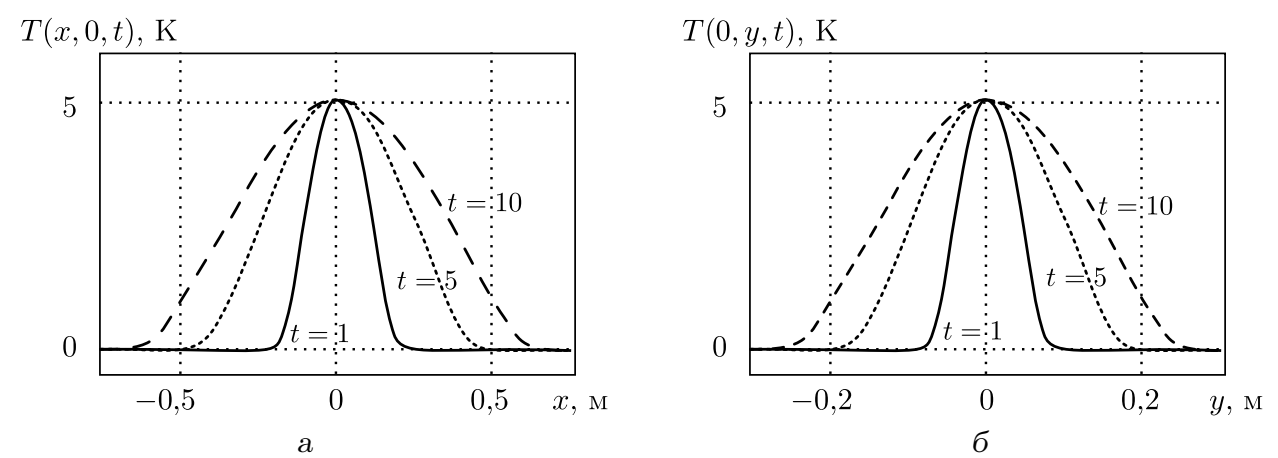

Рис. 1. Распределение температур при $\sigma=0,5:$ a- сечение $y=0, \sigma$-сечение $x=0$ 

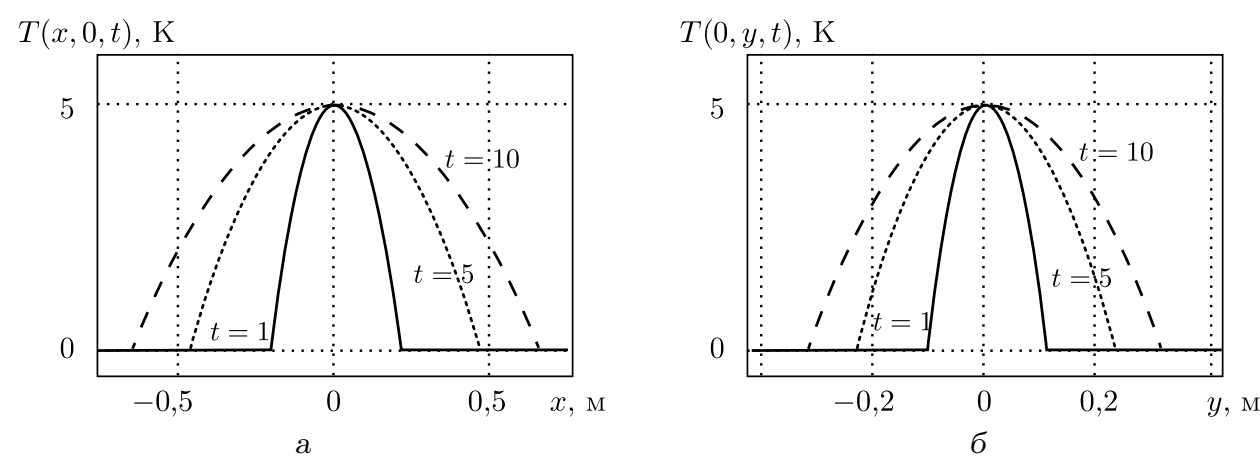

Рис. 2. Распределение температур при $\sigma=1$ : $\mathrm{a}$ - сечение $y=0, \sigma$ - сечение $x=0$
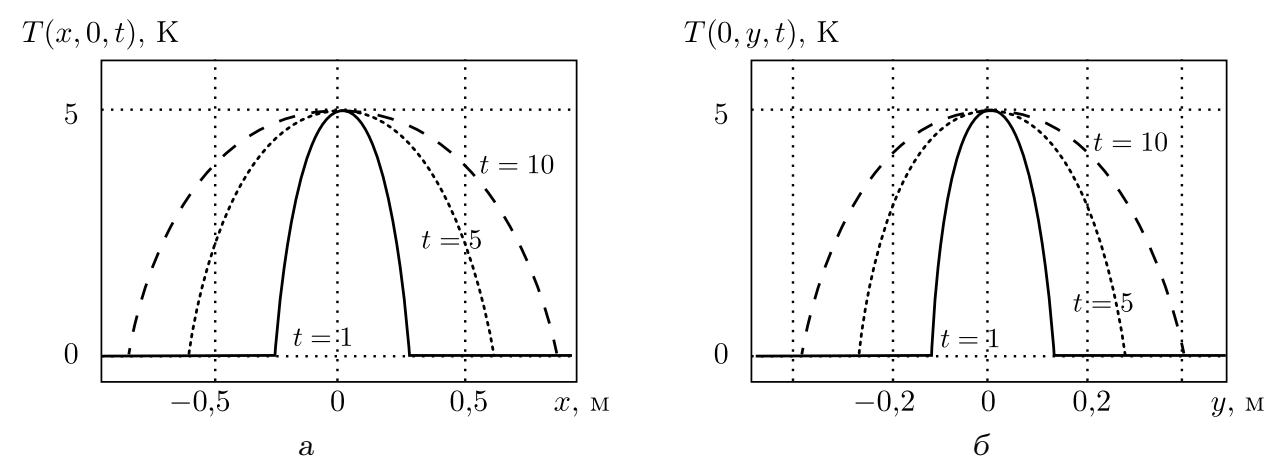

Рис. 3. Распределение температур при $\sigma=1:$ - сечение $y=0, \sigma$ - сечение $x=0$

фронт тепловой волны распространяется со скоростями $V_{x}=\sqrt{T_{0}^{\sigma} \frac{a}{\sigma} \frac{k_{\xi}}{b t}}$ вдоль оси $O x$, $V_{y}=\sqrt{T_{0}^{\sigma} \frac{a}{\sigma} \frac{k_{\eta}}{b t}}$ вдоль оси $O y$.

Компоненты вектора теплового потока вычисляются по формулам

$$
q_{x}=\lambda_{x x} \frac{\partial T}{\partial x}+\lambda_{x y} \frac{\partial T}{\partial y}, \quad q_{y}=\lambda_{y x} \frac{\partial T}{\partial x}+\lambda_{y y} \frac{\partial T}{\partial y} .
$$

В соответствии с (17) компонента теплового потока $q_{x}$ по оси $O x$ будет определяться выражением

$$
q_{x}(x, 0, t)=\lambda_{x x} \frac{1}{\sigma}\left(T_{0}^{\sigma}-\frac{\sigma b}{4 a t k_{\xi}} x^{2}\right)^{\frac{1-\sigma}{\sigma}}\left(-\frac{\sigma b}{2 a t k_{\xi}} x\right) .
$$

При $0<\sigma<1$ тепловой поток на границе фронта равен нулю. При $\sigma>1$ тепловой поток на границе фронта не существует. При увеличении параметра $\sigma$ поверхности, образуемые изотермами, не равными нулю, имеют большую область определения.

В точках фронта при $\sigma \geqslant 1$ первые производные по времени и первые и вторые производные по пространственным переменным не существуют.

При $0,5 \leqslant \sigma<1$ первые производные по времени и по пространству существуют внутри областей, ограниченных эллипсами с полуосями $l_{x}, l_{y}$, и в точках фронта. При $0,5<\sigma<1$ вторые производные в точках фронта не существуют.

При $0<\sigma<0,5$ существуют производные по времени и вторые производные по пространственным переменным внутри областей, ограниченных эллипсами с полуосями $l_{x}, l_{y}$, и в точках фронта. 
Выводы. Впервые получено и проанализировано аналитическое решение краевой задачи в нелинейном анизотропном пространстве для квазилинейного уравнения теплопроводности с тензором теплопроводности, компоненты которого являются степенными функциями температуры. Это решение показало волновой характер распространения тепла. Волновыми фронтами в анизотропном случае в различные моменты времени являются эллипсы на плоскости.

Проведён анализ существования решения на фронте волны и производных разных порядков по времени и пространству на всей плоскости в зависимости от показателя степени температуры, от которой зависят компоненты тензора теплопроводности.

Работа выполнена при поддержке РФФИ (проекты 08-08-00880-a, 08-08-00896-а) и Президента Российской Федерачии (проекты МK-646.2008.8, MK-1184.2009.8, MK-1669. 2009.8).

\section{БИБЛИОГРАФИЧЕСКИЙ СПИСОК}

1. Кудинов В.А. Анализ нелинейной теплопроводности на основе определения фронта температурного возмущения // Теплобизика высоких температур, 2006. - Т. 44, № 5. C. $577-585$.

2. Лыков А. В. Теория теплопроводности. - М.: Высш. шк., 1967. - 600 с.

3. Шашков А.Г., Бубнов В. А., Яновский С. Ю. Волновые явления теплопроводности. М.: Эдиториал УРСС, 2004. - 290 с.

4. Самарский А.А., Галактионов В.А., Курдюмов С. П., Михайлов А. П. Режимы с обострением в задачах для квазилинейных параболических уравнений. - М.: Наука, 1987. $478 \mathrm{c}$.

5. Соболев С.Л. Процессы переноса и бегущие волны в локально-неравновесных системax // УФH, 1991. - Т. 161, № 3. - С. 5-29.

6. Формалёв В. Ф. Тепломассоперенос в анизотропных телах. Обзор // Теплофизика высоких температур, 2001. - Т. 39, № 5. - С. 810-832.

Поступила в редакцию $11 / \mathrm{X} / 2009$; в окончательном варианте - 15/III/2010.

\section{MSC: 80A17, 80M25}

\section{SIMULATION OF HEAT WAVES IN AN NONLINEAR ANISOTROPIC SPACE}

\section{F. Formalyov, I. A. Selin, K. L. Kuznetcova}

Moscow Aviation Institute (State University of Aerospace Technologies), 4, Volokolamskoe Shosse, Moscow, 125993.

E-mails: sollo6@bk.ru, priem@mai.ru

For the first time analytical solution of the problem with boundary conditions in the non-linear anisotropic space for the quasilinear parabolic heat equation where heat conductivity tensor's components are temperature functions is obtained. This solution has shown a wave type of the heat diffusion. Wave's fronts are ellipse in the anisotropic space at different time moments. Existence of the solution in wave's front and time derivative and space derivatives different orders has been analysed dependence from power of temperature which influences heat conductivity tensor's components.

Key words: quasilinear parabolic equation, heat wave, heat conductivity tensor, temperature, heat flow, front.

Original article submitted $11 / \mathrm{X} / 2009$; revision submitted 15/III/2010.

Vladimir F. Formalyov (Dr. Sci. (Phys. \& Math.)), Professor, Dept. of Computational Mathematics \& Programming. Iliya A. Selin, Postgraduate Student, Dept. of Computational Mathematics \& Programming. Ekaterina L. Kuznetcova (Ph. D. (Phys. \& Math.)), Doctoral Candidate, Dept. of Computational Mathematics \& Programming. 